\title{
Regeneration of sutured human peripheral nerves: an electrophysiological study
}

\author{
RAFAELS. DONOSO, JOHN P. BALLANT Y NE, \\ A N D S T I G H A NSE N \\ From Glasgow University Department of Neurology and Division of Clinical Physics, \\ Institute of Neurological Sciences, Southern General Hospital, Glasgow
}

\begin{abstract}
S UMMARY Electrophysiological and clinical assessment of recovery of function was undertaken on 34 median and 33 ulnar nerves which had been resutured after complete section three and a half months to 24 years previously. An evaluation of different methods of repair was attempted. Our results suggested that re-exploration of the site of suture is indicated in the absence of voluntary activity on needle EMG by seven months (12 months for grafts), of an electrically evoked muscle action potential, measurable distal motor latency, or motor nerve conduction velocity by 10 months (14 months for grafts), or of clinically detectable voluntary muscle movement by 10 months after suture. By present techniques of repair useful prognostic information cannot be obtained by a consideration of sensory parameters either clinical or electrophysiological.
\end{abstract}

Peripheral nerve injuries, accidental or selfinduced, pose an increasing problem in our modern mechanised society. Resuture of sectioned human peripheral nerves is widely practised using various techniques including primary and secondary suture, fascicular suture, and nerve grafts. Quantitative assessment of recovery of function in nerves so repaired presents considerable difficulties. Prognostic indices of successful outcome of the repair procedures are poorly defined, and the merits of various surgical techniques remain controversial. While a number of electrophysiological studies on peripheral nerve regeneration have been undertaken in animals (Berry et al., 1944; Cragg and Thomas, 1964; Jacobson and Guth, 1965; Dickson et al., 1977), only a few such studies have been performed in man (Struppler and Huckauf, 1962; Ballantyne and Campbell, 1973). Early signs of muscle reinnervation can be detected by needle electromyography (Kline et al., 1969). Quantitation of the amount of reinnervation in terms of the EMG activity has been attempted on this basis (Grabb, 1968; Dolenc and Janko, 1976). In an earlier report (Ballantyne and Campbell, 1973) we presented electrophysiological observations on 20

Address for correspondence: Dr John P. Ballantyne, Department of Neurology, Institute of Neurological Sciences, Southern General Hospital, Glasgow GS1 4TF.

Accepted 22 August 1978 patients who had sustained median or ulnar nerve section repaired by resuture. The present investigation on a further 43 cases of sectioned and resutured nerves has been added to the original study, giving a total of 67 operated nerves. The purpose of the present paper is to present the results of our combined observations on these 67 operated nerves in terms of the quality of electrophysiological recovery related, where feasible, to the technique of resuture, the time over which continued recovery occurs, and to quantify the degree of that recovery both clinically and electrophysiologically.

\section{Methods}

Fastest motor nerve conduction velocities (FMNCV) and shortest distal motor latencies (SDML) were measured in ulnar and median nerves using an established technique (Hodes et al., 1948), the evoked muscle response being recorded from surface electrodes over the abductor digit minimi (ADM) or abductor pollicis brevis (APB) muscles or both. Stimulation was via saline-soaked, gauze-padded, silver electrodes fixed in a Perspex holder $30 \mathrm{~mm}$ apart and placed over the median or ulnar nerve at the wrist and held in position by an elasticated strap. Proximal stimulation of the nerve trunk was via a hand-held 
electrode with similar properties to that used for distal stimulation. Stimuli were delivered at one stimulus per three seconds from the isolated stimulator of the Hewlett Packard electromyograph (Type 1510A), and the evoked muscle potentials were displayed on its variable persistence oscilloscope. All stimulus intensities were $20 \%$ supramaximal. Single stimulus pulses of $0.1 \mathrm{~ms}$ duration were used in most cases, exceptionally pulses of up to $0.3 \mathrm{~ms}$ duration were required to obtain a response.

Orthodromically evoked sensory nerve action potentials (SNAP) were recorded from the surface electrodes over the ulnar or medial nerve at the wrist used for motor stimulation. Sensory nerve action potentials were evoked by stimulation of the index or little fingers respectively for median and ulnar nerve studies. Sensory stimulating electrodes consisted of silver strips of $5 \mathrm{~mm}$ width and $60 \mathrm{~mm}$ length coated in electrode jelly and secured at the base and near the middle phalanx of the respective fingers-that is, approximately $30 \mathrm{~mm}$ apart. The stimuli were delivered at one pulse per three seconds, each pulse being of 0.05 ms duration. Stimulus intensities were $20 \%$ supramaximal. The orthodromically evoked SNAPs over the median and ulnar nerves at the wrist were displayed on the Hewlett Packard variable persistence oscilloscope on which manual measurements of latency and amplitude were obtained. In a few cases an averaging technique was employed for sensory nerve action potentials using a Medelec electromyograph.

Needle electromyography was undertaken in either the ADM or the APB muscles. Some cases were supplemented by similar studies in the median or ulnar innervated heads of the flexor digitorum muscle where nerve section had occurred proximally in the forearm. All electrophysiological measurements were undertaken at an arm temperature of $32-34^{\circ} \mathrm{C}$.

Motor power was assessed in the small hand muscles on the MRC scale (Medical Research Council, 1954) using APB for median nerve and ADM for ulnar nerve lesions. A clinical sensory evaluation of pinprick, light touch, and two point discrimination was undertaken in most patients. For pinprick and light touch the patients' sensation was graded present or absent, normal or abnormal, and no further quantitation was attempted. In this study two point discrimination was assessed as the smallest separation of the calipers at which the two points could be appreciated by the patient in the affected nerve territory.

In all cases the median and ulnar nerves in the normal unaffected upper limb were used as controls for both the electrophysiological and clinical observations. The electrophysiological parameters were expressed as the percentage ratio of the value for the affected side to the value for the normal side. A separate control group was studied to determine the $95 \%$ confidence limits for these ratios.

\section{Subjects}

There were 63 patients aged 8-65 years (mean $31.7 \pm 14$ years). Thirty-two of these patients had sustained complete ulnar nerve section and suture undertaken four months to 10 years before the present study. Eight of these patients had sustained section of the ulnar nerve in the forearm and the remainder at the wrist. Thirty-four of the patients had complete division of the median nerve repaired three and a half months to 24 years before the present study. In four of these patients the site of the section was in the forearm and in the others at the wrist. In the patients with median nerve lesions, 27 repairs had been undertaken by primary suture, four by delayed suture, and three by grafting. In the ulnar nerve group, 17 nerves were repaired by primary suture, eight by delayed suture, and seven had been grafted. Delayed suture was more common in those patients who had been treated more than four years before the present study. A few patients whose injuries had occurred within the past four years had delayed suture. This had been undertaken because of failure of the primary repair. Six nerves (three median, three ulnar) were repaired by the fascicular method.

\section{Results}

\section{NEEDLE ELECTROMYOGRAPHY}

Needle electromyography was undertaken in 16 APB muscles (median nerve lesions) and 19 ADM muscles (ulnar nerve lesions). The studies were undertaken from four months to 24 years after the time of surgery. Eight patients were examined within five months of the nerve repair. Of these, three were primary fascicular repairs and all showed voluntary motor unit activity at that time. Of the five remaining nerves studied at that time only one showed evidence of voluntary motor unit activity. From seven months after operation onwards, however, muscles supplied by all nerves that had undergone primary repair showed voluntary motor units with the exception of one grafted nerve studied at nine months postoperatively in which there was no spontaneous electrical activity.

In general, the amplitude of the motor unit 
potentials (MUP) increased with the passage of time from operation (Fig. 1). In a few patients high amplitude motor unit potentials were present in early post-repair recordings. For example, in the two nerves repaired by fascicular suture studied at five months, MUPs of 4-5 mV were seen. Patient JF at nine months had MUPs of $5 \mathrm{mV}$ amplitude but was found to have a Martin Gruber anastomosis. Patient DS at eight months also had MUPs of $5 \mathrm{mV}$, but had aberrant innervation from the ulnar nerve to the thenar muscles. A third patient (BK) had MUPs of amplitude up to $10 \mathrm{mV}$ at 10 months. In this one patient, however, we had undertaken needle electromyography in the first dorsal interosseous muscle, and at the time of operation a median motor branch to the first lumbrical muscle had been divided and implanted into the adductor pollicis brevis muscle. It is possible that reinnervation of the first dorsal interosseous muscle may have occurred at an early stage via this branch. In all the patients with primary suture showing high amplitude MUPs before 10 months some form of anomalous inner- vation was present except in the cases repaired by fascicular suture.

\section{MOTOR CONDUCTION VELOCITY}

Fastest motor nerve conduction velocities were studied in 60 nerves, 28 median and 32 ulnar (Fig. 2). Motor nerve conduction first became apparent at five months in three nerves all repaired by the fascicular method, and all had conduction velocities comparable to the normal side. However, of the 19 nerves studied before 10 months after suture, only eight $(42 \%)$ had detectable motor nerve conduction. Between 10 and 20 months, however, FMNCVs were obtainable in $90 \%$ of nerves. Thereafter FMNCV was measurable in all nerves investigated.

\section{SHORTEST DISTAL MOTOR LATENCY}

Shortest distal motor latencies were studied in 62 nerves, 32 ulnar and 30 median (Fig. 3). Before 10 months only eight of 19 patients had recordable SDMLs, but between 10 and 20 months, 17 of 18 patients had measurable values although only two

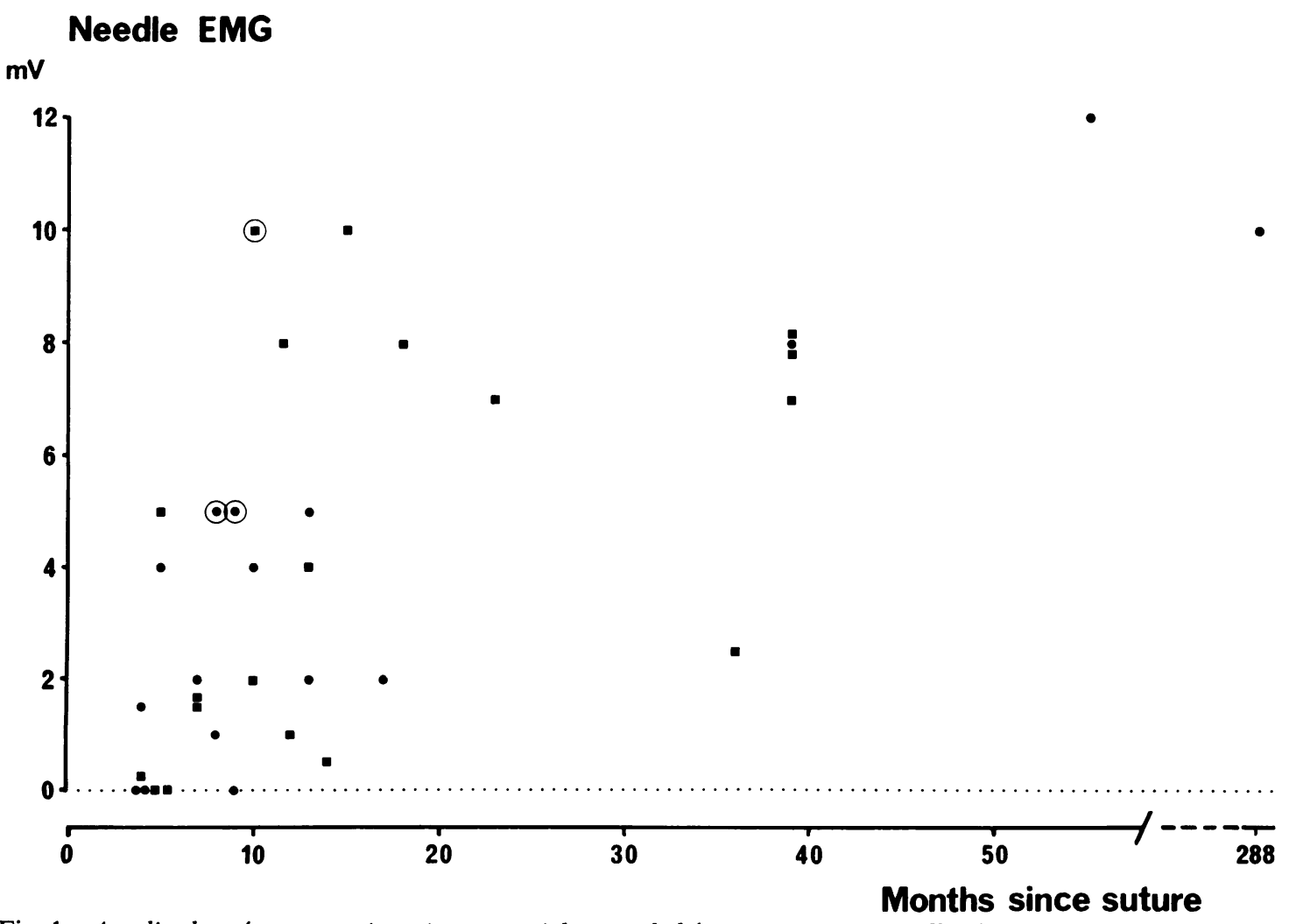

Fig. 1 Amplitudes of motor unit action potentials recorded from concentric needle electrodes. $=a b d u c t o r$ pollicis brevis muscle; $\square=a b d u c t o r$ digiti minimi muscle; circles indicate anomalous innervation. 


\section{\% FMNCV}

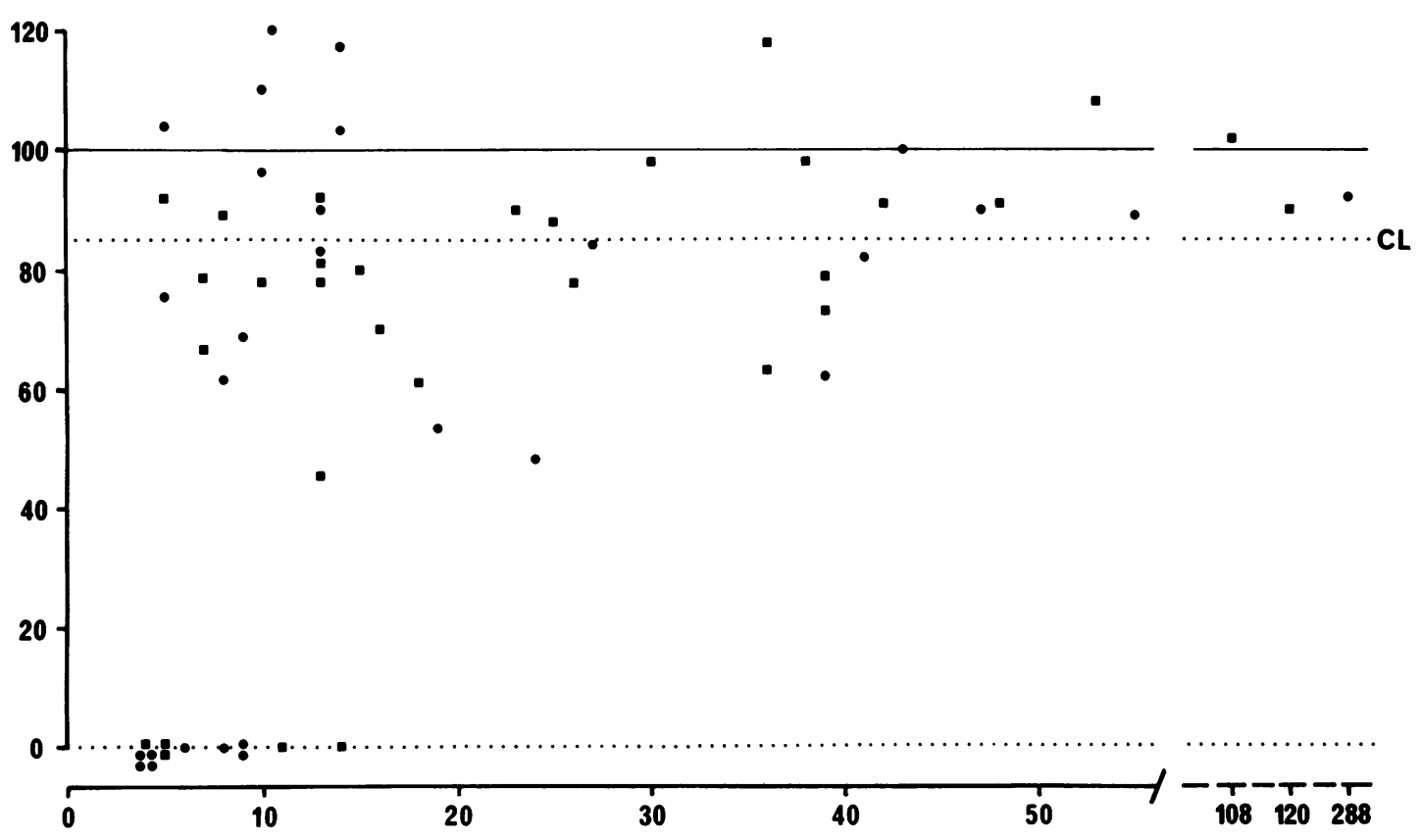

\section{Months since suture}

Fig. 2 Fastest motor nerve conduction velocities as percentage of the value from the normal contralateral nerve. $\bullet=$ median nerve; $\square=$ ulnar nerve; $C L=$ lower $95 \%$ confidence limit for normal subjects.

fell within the $95 \%$ confidence limits of the normal side. It is apparent from Fig. 3 that there is a steady improvement in SDML with time, and from 40 months onwards only three of nine nerves had SDMLs outwith the upper limit of normal.

\section{MOTOR POWER}

Power was evaluated on the MRC scale $0-5$ in the small muscles of the hand in 29 ulnar and 27 median operated nerves (Fig. 4). Before 10 months there was no detectable power in the abductor digiti minimi or the abductor pollicis brevis muscles in $54 \%$ of patients, $23 \%$ had grade $3-4$ power in these muscles, and a further $23 \%$ grade 1-2 power. From 10 to 20 months, a grading better than 0 was present in the territories of all nerves examined, $80 \%$ lying between grades 3-4. At more than 40 months after repair, $83 \%$ of patients had grade 4 power or better. There is, therefore, a steady improvement in muscle power with the passage of time from suture. The amplitudes of the supramaximally evoked muscle action potentials (SEMAP) recorded from surface electrodes over the ADM or APB muscles cor- related well with the clinical assessment of power in the same muscles (Fig. 5), coefficient of rank correlation, Spearman's rho $=0.84 ; \mathrm{P}<0.0001$ ).

\section{SENSORY NERVE ACTION POTENTIALS}

Sensory nerve action potentials (Fig. 6) were studied in 63 nerves, 33 median and 30 ulnar, and were detectable in only five of 39 nerves $(13 \%)$ during the first 20 months after suture. However, from 20 months onwards, 13 of 24 nerves $(54 \%)$ had detectable sensory nerve action potentials. The percentage recovery in the two groups is significantly different (chi squared $=10.5 ; \mathrm{P}<0.005$ ). Nevertheless SNAPs could not be detected in two ulnar nerves operated nine and 10 years previously despite the good recovery of motor function. Once detectable there was no tendency for the amplitude of the SNAP to increase with the passage of time, and in only three of the 63 nerves studied did the SNAP amplitudes exceed the $95 \%$ confidence limits of normal. Most SNAPs had an amplitude between 10 and $40 \%$ of control values. The two patients with the earliest recorded SNAPs at five and eight months after suture respectively had 
$\%$ SDML

$\infty-$ s.?.:.

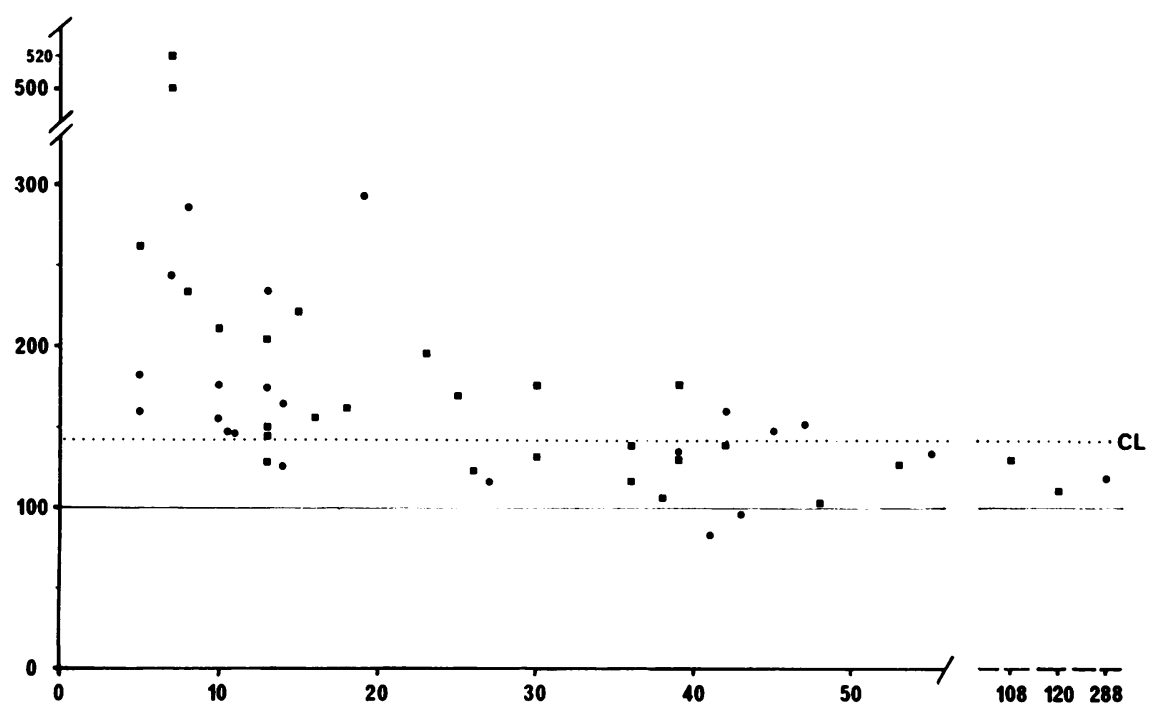

Months since suture

Fig. 3 Shortest distal motor latency as percentage of the value from the normal contralateral nerve. $\bigcirc=$ median nerve, $\square=$ ulnar nerve; $C L=$ upper $95 \%$ confidence limit for normal subjects.

both had primary fascicular repair of the ulnar nerve at the wrist.

\section{CLINICAL ASSESSMENT OF SENSATION}

Clinical assessment was undertaken in 57 nerves, 31 median and 26 ulnar. In keeping with the electrophysiological recovery in sensory nerves, the clinical investigation yielded equally poor results. In no patient was touch or pain sensation subjectively normal but these were not quantified further. Two point discrimination was quantified as the smallest separation of the points that could be recognised by the patient. In no patient was this within the normal range, nor was there any evidence of a tendency for improvement with the passage of time.

\section{Discussion}

\section{NEEDLE ELECTROMYOGRAPHY}

Voluntary motor unit activity in the territories of the denervated nerves was first detected at four months after suture in two patients (one primary and one fascicular repair). With the exception of grafted nerves, all resutured nerves showed EMG activity in the target muscles by seven months, in agreement with the report of Sunderland (1968).
In grafted nerves EMG activity was in general delayed, but was detected in all such nerve territories by 14 months. Forearm lesions did not appear to behave differently from more distal lesions, EMG activity being detected in one such nerve at four months.

In general the amplitudes of the voluntarily evoked MUPs increased with time after repair (Fig. 1), but in a few cases high amplitude MUPs were found in the early months. Since motor reinnervation depends upon regrowth of axons and collateral sprouting by those axons (Simpson, 1970), the demonstration of large potentials at an early stage raises the probability of reinnervation from undamaged and aberrant motor axons. Such anomalies of innervation have been described (Mannerfelt, 1966; Seddon, 1972) and can be detected by motor nerve stimulation. Three of our patients with large MUPs at an early stage were found to have anomalies of innervation to the hand muscles but this was not the case in the patients with fascicular repair who had high amplitude MUPs.

SHORTEST DISTAL MOTOR LATENCIES AND FASTEST MOTOR NERVE CONDUCTION VELOCITIES

The shortest distal motor latencies showed a pro- 


\section{Muscle power \\ MRC grading}
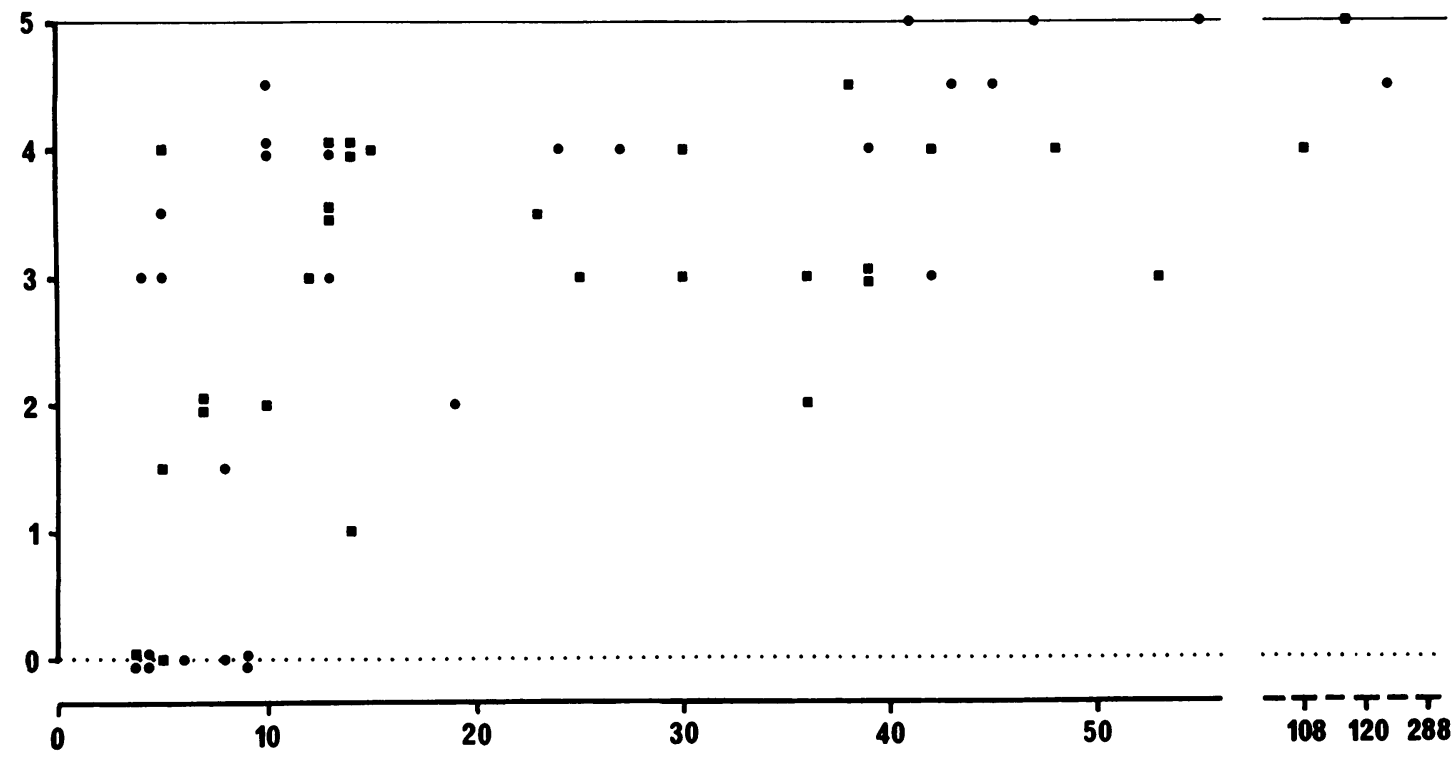

Months since suture

Fig. 4 Muscle power (MRC grading).

= abductor pollicis brevis muscle;

=abductor digiti minimi muscle.

Muscle power

MRC grading

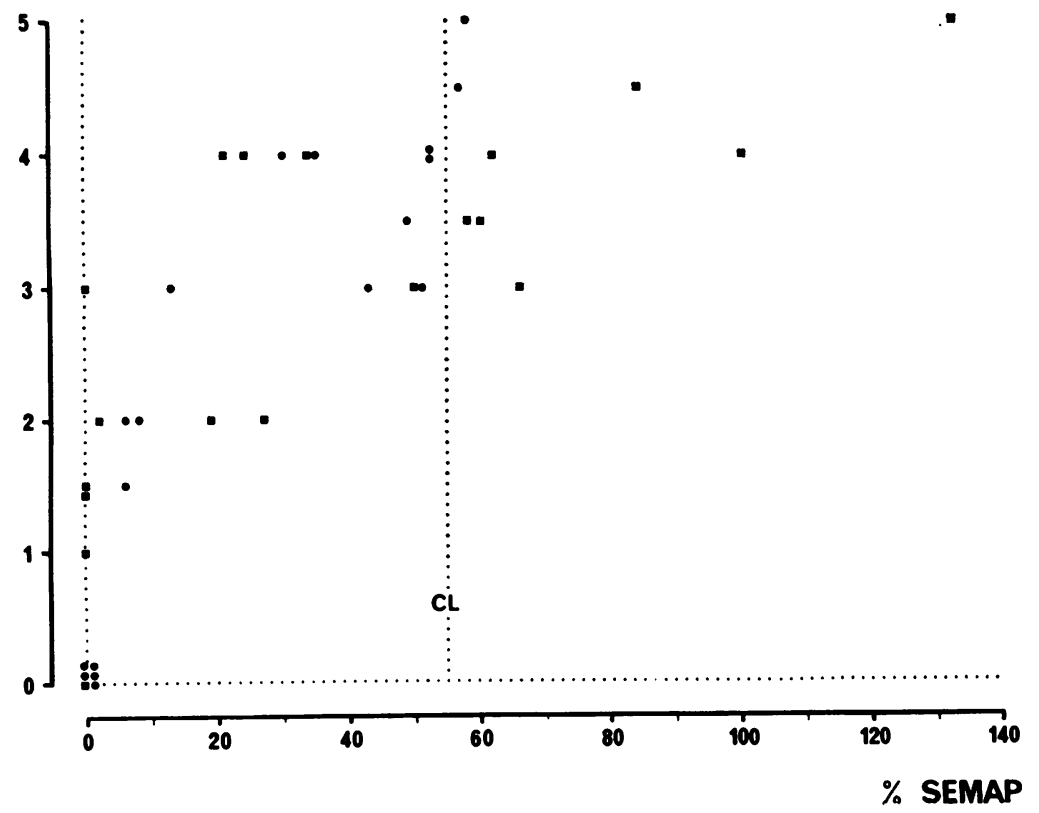

Fig. 5 Relationship between muscle power (MRC grading) and supramaximally evoked muscle action potential (as percentage of the value from the normal contralateral muscle). There is a significant positive correlation (coefficient of rank correlation, Spearman's rho $=0.84 ; P<0.0001)$. abductor pollicis brevis muscle; $=$ abductor digiti minimi muscle; $C L=$ lower $95 \%$ confidence limit for normal subjects. 


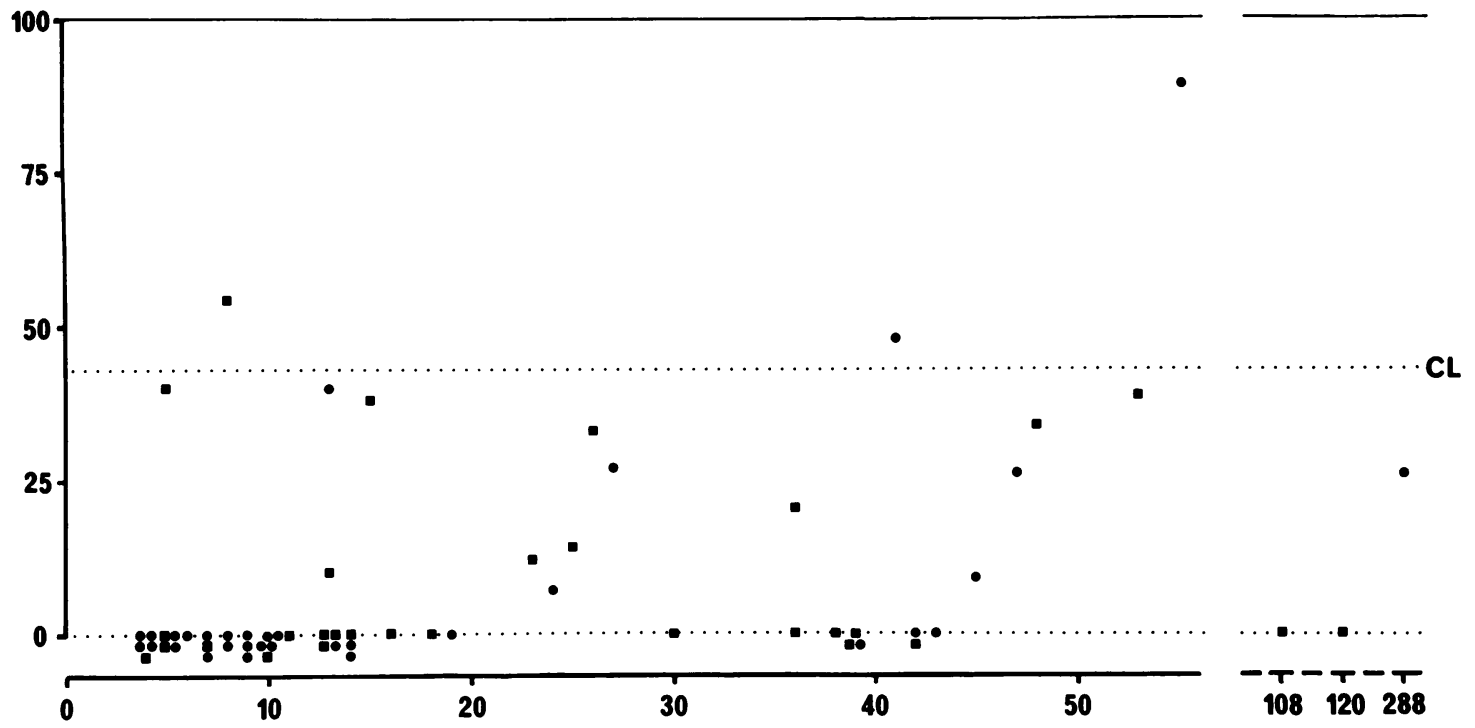

Months since suture

Fig. 6 Orthodromic sensory nerve action potentials as percentage of the value from the contralateral nerve. $\bullet=$ median nerve; $\square=$ ulnar nerve; $C L=$ lower $95 \%$ confidence limit for normal subjects.

gressive reduction with the passage of time but in only two of the nerves tested before 20 months after suture did values fall within the $95 \%$ confidence limits of the control nerves. In regenerating nerve fibres the SDML reflects the conduction in regenerating motor axons which have grown distally from the site of suture and made functional contact with the muscle fibres together with a component caused by the neuromuscular delay. Since the stigmatic surface recording electrode was placed over the motor point of the muscle, there was no additional delay from conduction of muscle fibre action potentials from the endplate zone to the electrode. This may contribute to the SDML if concentric needle electrodes are used to record the muscle action potentials.

Histological studies have shown that there is a progressive increase in the axonal diameter of regenerating fibres (Berry et al., 1944). In sectioned and resutured rabbit nerves, Cragg and Thomas (1964) reported that axonal diameter was $90 \%$ of normal by 16 months. Schröder (1972) reported a mean axonal diameter of $79 \%$ in regenerating axons in the sciatic nerve of the dog at 12 months, but in follow-up studies from 12-24 months, no further increase in mean fibre diameter occurred. The steady improvement in the SDMLs in regener- ating nerves in this study can, therefore, be attributed in part to maturational changes leading to increase in the diameters and conduction velocities of the regenerating axons with time. When animal experiments have shown axonal diameters ranging from $79-90 \%$ of normal by $12-16$ months, SDMLs in our patients remained quite grossly elevated. This discrepancy can no doubt be explained in terms of defective neuromuscular transmission in the regenerating nerve fibres as reported by Hodes et al. (1948) and Dennis and Miledi (1971). The studies of Hakelius and Stålberg (1974) also indicate that conduction in collateral sprouts of intramuscular nerve fibres may remain defective for some months, which further increases the SDML. The fact, however, that most of the SDMLs reach the normal range by 40 months and above must indicate that axonal diameter in these regenerating nerve fibres eventually approaches, and indeed reaches, normal in most patients, provided, of course, that the relationship between axonal diameter and conduction velocity is maintained in these regenerating nerve fibres. A slow increase in the axonal diameter over the years must be considered a possibility in these nerves, and such a hypothesis would not be negated by the results obtained from animal experimentation in 
view of the difficulties of long-term follow-up of such preparations.

Motor nerve conduction was first measurable at five months and was found in four of 12 nerves four to eight months after repair, nine of 12 nerves eight to 12 months after repair, and 12 of 15 nerves 10-20 months after repair. With two of the three grafted nerves, no evoked potential could be detected in the muscle at 10-20 months. At 20 months or longer after repair all nerves had measurable FMNCVs. A moderate but progressive increase in FMNCV occurs with time (Fig. 2), with a rise in the mean motor nerve conduction velocity from $71 \%$ of control nerves at $10-20$ months, $85 \%$ of control nerves at $20-40$ months, and $91 \%$ of control nerves at $40-55$ months. From 42 months after repair onwards all motor nerve conduction velocities were within the $95 \%$ confidence limits of normal. In nerves studied from four months onwards, however, there were many which had motor nerve conduction velocities within the normal range. The lowest values showed a steady improvement with time. This presumably reflects the randomness of the reinnervation process, from large diameter axons in those cases with early normal values for motor nerve conduction whether due to technique of repair or chance. In the more slowly conducting nerves, however, smaller diameter axons have presumably been first and in the greatest numbers to reinnervate the target muscle. None of the conduction velocities recorded in the regenerating nerves fell below $50 \%$ of normal values, representing an absolute conduction velocity in the region of $25-30 \mathrm{~m} / \mathrm{s}$. These values are at the lower limit of the range of conduction velocities for normal human motor fibres reported by Thomas et al. (1959). In so far as FMNCV is measured along the segment of the nerve proximal to the site of suture in the majority of patients, the progressive improvement in the lowest values of FMNCV could be caused either by the subsequent functional contact of regenerating sprouts from large diameter axons or by maturational changes in the existing smaller diameter motor axons above the site of suture. It appears unlikely that regenerating axons continue to innervate the muscle more than three years after suture (Bowden and Gutmann, 1944). The further increase in FMNCV proximal to the site of suture cannot, therefore, be explained by further increase in the number of motor axons making functional contact with the muscle. Rather, this increase in conduction velocity must reflect physiological changes occurring in the proximal axons. It has been reported that larger diameter axons innervate muscle units that contain a greater number of muscle fibres than do smaller diameter motor axons (Henneman et al., 1965). It has also been demonstrated that the ultimate diameter of a regenerated nerve fibre is influenced considerably by whether or not it makes functional contact with an end organ. Fibres that make no functional contact remain of small diameter while motor fibres connected to sensory terminals are of larger diameter than those without a peripheral connection, but not as large as those that reinnervate a muscle (Allt, 1976). After nerve section and resuture there is a permanent reduction in the number of axons innervating the target muscle, leading to an increase in the muscle fibre content and therefore peripheral loading of these axons. We have found evidence of this in the high amplitude MUPs noted in our reinnervated muscles. It remains possible, therefore, that the increase in peripheral loading of the reinnervating axons leads to a progressive increase in axonal diameter such that the relationship of axonal diameter to muscle fibre content of the motor unit tends to approach normal. Furthermore, in the previous study (Ballantyne and Campbell, 1973) a steady increase in the amplitude of the mixed nerve action potential (stimulating and recording above the site of suture) was noted in human nerves over a similar period of time. A steady increase in the axonal diameter would also explain that observation.

\section{MOTOR POWER}

From 10 months onwards all patients had detectable function in the denervated muscles that could be graded on the MRC scale (Fig. 4). By 40 months after suture, $80 \%$ of muscles showed grade 4 power or more, a result which is considerably better than that reported by Nicholson and Seddon (1957) who noted that $30 \%$ of subjects had grade 4 power or more by 36 months. The improvement in power was accompanied by a progressive increase in the amplitude of the SEMAP from the target muscle. These parameters show a significant positive correlation. We found this correlation to be of practical value in patients who had adopted "trick" manoeuvres to compensate for the loss of power in the muscles. In these patients the SEMAP was small, but MRC grading was relatively good. $\mathrm{Re}$-examination of the patients uncovered the use of other muscles to overcome the disability, to the deception of the clinical assessor on the first examination. On the basis of this experience we feel that the SEMAP is a better index of return of function in the denervated muscle than clinical assessment on the MRC scale. Providing the recording is made from surface electrodes over the motor point of the muscle, the technique is reproducible. 


\section{SENSORY FUNCTION}

While there is a definite increase in the number of nerves from which a SNAP can be recorded with the passage of time, there is no obvious improvement in the amplitudes of the evoked responses with time (Fig. 6). Nevertheless, throughout the course of this study, at nine years in one patient, 10 years in another, and between 20 and 40 months after suture no SNAP could be evoked in a considerable number of nerves. Before 10 months, SNAPs were detected in only two nerves, both of which had been repaired by the fascicular method. Up to 20 months after repair only $13 \%$ of nerves had a measurable SNAP. From 20 to 40 months the SNAP is seen in $50 \%$ of nerves, and in $75 \%$ between 40 and 55 months. When compared with the clinical and electrophysiological recovery in motor nerve fibres, the results in the sensory fibres appear disappointing. The apparent discrepancy in recovery, however, is likely to be more apparent than real. The amplitude of the SNAP is determined by the number of conducting axons and the synchronisation of their responses under the recording electrodes. When the number of conducting axons falls below a certain value, or their action potentials become dispersed because of slowing of conduction, or both, then no SNAP may be recordable. A comparable sequence of events in the motor nerve may still be accompanied by a discernible or even large muscle action potential on stimulation of the motor nerve. Relatively few motor nerve fibres innervating greatly enlarged motor units may give rise to a relatively large muscle action potential which can be easily detected by surface recording electrodes.

In keeping with the electrophysiological results, clinical recovery of sensation was also poor. In none of the nerve territories examined was touch or pain sensation normal. In our earlier study (Ballantyne and Campbell, 1973) two point discrimination was found to be no better than chance In the remaining 45 nerves examined in the present investigation sensibility to two points simultaneously applied to the appropriate finger pulp was quantified in terms of the smallest separation of the two points that could be consistently recognised as such by the patient. In only six of the 45 nerves was two point discrimination present by these criteria, but in none was it within the normally accepted range (less than $5 \mathrm{~mm}$ ). The values obtained were $6,25,25,25,30$, and $40 \mathrm{~mm}$. There was no correlation between these values and the amplitudes of the sensory nerve action potentials. Indeed, in only two of the sensory territories was a SNAP detected when the value for two point discrimination was 6 and $40 \mathrm{~mm}$ respectively.

\section{Conclusions}

In regenerating resutured human nerves, voluntary motor unit activity becomes apparent at four months and is present in all patients by seven months after suture (grafted nerves 12 months after suture). The amplitudes of the voluntarily evoked MUPs increase steadily with time, and can attain giant unit dimensions. The detection of high amplitude MUPs in the early months of regeneration may indicate the presence of anomalous innervation.

Using surface electrodes over the target muscle SDML and FMNCV should be measurable by 10 months after suture (14 months for grafts and forearm lesions).

Voluntary motor activity should be detectable by 10 months after suture, grade 3 power should be present by 40 months, and, grade 2 or better by 20 months. Muscle power continues to improve to 55 months and possibly longer. The amplitude of the SEMAP recorded from surface electrodes over the target muscle is a better quantitative index of recovery of muscle function than clinical assessment of power using the MRC scale.

Both clinical and electrophysiological recovery in sensory nerve fibres are poor. Normal sensation to touch, pain, and two point discrimination was not found in any nerve territory even up to 24 years after suture. Complete absence of recordable SNAPs was found as late as 10 years after suture.

There is no clear difference in the electrophysiological or clinical recovery in lesions in the forearm compared to wrist, in median or ulnar lesions over the long-term, nor in the long-term results of primary suture, secondary suture, or grafting despite the initial delay in the appearance of signs of recovery in the latter.

While the number of nerves repaired by the fascicular method was small (six nerves), the results are encouraging. These nerves showed the earliest and in general the best recovery in the early stages. This method of repair had been undertaken in a relatively young group of patients, and younger subjects are known to, in general, have better recovery than older age groups (Nicholson and Seddon, 1957). The results nevertheless indicate that this method of repair merits further study.

In the early assessment of recovery of function in a sectioned and resutured nerve, our investigations suggest that re-exploration of the site of suture is indicated in the absence of voluntary activity on needle EMG by seven months (12 months for grafts), of an electrically evoked muscle action potential, measurable distal motor 
latency or motor nerve conduction velocity by 10 months (14 months for grafts), or of clinically detectable voluntary muscle movement by 10 months after suture. By present techniques of repair (with the possible exception of the fascicular type) useful prognostic information cannot be obtained by a consideration of sensory parameters either clinical or electrophysiological.

We wish to thank Mr J. C. Semple and colleagues for referring most of the cases. We are grateful to Professor J. A. Simpson for his advice and for use of the facilities in his department. Our thanks are due also to Dr Andrew Weir for his assistance, and to Miss J. Weston for secretarial services. RSD held a British Council Scholarship and SH was supported by a grant from the Wellcome Trust.

\section{References}

Allt, G. (1976). Pathology of the peripheral nerve. In The Peripheral Nerve, pp. 666-739. Edited by D. N. Landow. Chapman and Hall: London.

Ballantyne, J. P., and Campbell, M. J. (1973). Electrophysiological study after surgical repair of sectioned human peripheral nerves. Journal of Neurology, Neurosurgery, and Psychiatry, 36, 797-805.

Berry, C. M., Grundfest, H., and Hinsey, J. C. (1944). The electrical activity of regenerating nerves in cat. Journal of Neurophysiology, 7, 103-115.

Bowden, R. E. M., and Gutmann, E. (1944). Denervation and re-innervation of human voluntary muscle. Brain, 67, 273-313.

Cragg, B. G., and Thomas, P. K. (1964). The conduction velocity of regenerated peripheral nerve fibres. Journal of Physiology, 171, 164-175.

Dennis, M., and Miledi, R. (1971). Lack of correspondence between the amplitudes of spontaneous potentials and unit potentials evoked by nerve impulses at regenerating neuromuscular junctions. Nature, New Biology, 232, 126-128.

Dickson, R. A., Dinley, J., Rushworth, G., and Colwin, A. (1977). Delayed (degenerate) interfascicular nerve grafting: a new concept in peripheral nerve repair. British Journal of Surgery, 64, 698-701.

Dolenc, V., and Janko, M. (1976). Nerve regeneration following primary nerve repair. Acta Neurochirurgica, 34, 223-234.

Grabb, W. C. (1968). Median and ulnar nerve suture. Journal of Bone and Joint Surgery, 50A, 964-972.
Hakelius, L., and Stålberg, E. (1974). Electromyographical studies of free autogenous muscle transplants in man. Scandinavian Journal of Plastic and Reconstructive Surgery, 8, 211-219.

Henneman, E., Somjen, G., and Carpenter, D. (1965). Functional significance of cell size in spinal motoneurons. Journal of Neurophysiology, 28, 560-580.

Hodes, R., Larrabee, M. G., and German, W. J. (1948). The human electromyogram in response to nerve stimulation and the conduction velocity of motor axons. Study on normal and injured peripheral nerves. Archives of Neurology and Psychiatry (Chicago), 60, 340-365.

Jacobson, S., and Guth, L. (1965). An electrophysiological study of the early stages of peripheral nerve regeneration. Experimental Neurology, 11, 48-60.

Kline, D. G., Hackett, E. R., and May, P. R. (1969). Evaluation of nerve injuries by evoked potentials and electromyography. Journal of Neurosurgery, 31, 128-136.

Mannerfelt, L. (1966). Studies on the hand in ulnar nerve paralysis. A clinical-experimental investigation in normal and anomalous innervation. Acta Orthopaedica Scandinavica, Supple. 87, 1-176.

Medical Research Council (1954). Peripheral Nerve Injuries. Special Report Series. Medical Research Council No. 282. Her Majesty's Stationery Office: London.

Nicholson, O. R., and Seddon, H. J. (1957). Nerve repair in civil practice: results of treatment of median and ulnar nerve lesions. British Medical Journal, 2, 1065-1071.

Schröder, J. M. (1972). Altered ratio between axon diameter and myelin sheath thickness in regenerated nerve fibres. Brain Research, 45, 49-65.

Seddon, H. J. (1972). Surgical Disorders of the Peripheral Nerves. Churchill-Livingstone: Edinburgh and London.

Simpson, J. A. (1970). Nerve injuries. General aspects. In Handbook of Clinical Neurology, vol. 7, chapter 7. Edited by P. J. Vinken and G. W. Bruyn. NorthHolland: Amsterdam.

Struppler, A., and Huckauf, H. (1962). Propagation velocity in regenerated motor nerve fibres. In Progress in Electromyography, pp 58-60. Edited by P. Pinelli. Elsevier: Amsterdam.

Sunderland, S. (1968). Nerves and Nerve Injuries. Livingstone: Edinburgh and London.

Thomas, P. K., Sears, T. A., and Gilliatt, R. W. (1959). The range of conduction velocity in normal motor nerve fibres to the small muscles of the hand and foot. Journal of Neurology, Neurosurgery, and Psychiatry, 22, 175-181. 